\title{
Genealogía del concepto de paz en el derecho internacional público
}

Genealogy of the concept of peace in international public law

\author{
Ana María Bonet de Viola ${ }^{1} \underline{\mathrm{ORCID}}$ \\ Rina Coassin ${ }^{2} \otimes \underline{O R C I D}$ \\ Elisabet Vidal ${ }^{3 凶} \underline{\mathrm{ORCID}}$ \\ Yael Selene Saidler ${ }^{4} \underline{\text { ORCID }}$
}

Fecha correspondencia:

Recibido: 14 de enero de 2020. Revisión: 23 de marzo de 2020. Aceptado: 24 de marzo de 2020.

\section{Forma de citar:}

Bonet de Viola, Ana María; Coassin, Rina; Vidal, Elisabet; Saidler, Yael Selene. "Genealogía del concepto de paz en el Derecho Internacional Público." Revista CES Derecho. Vol. 11 , No. 1, enero a junio de 2020, 147-156.

\section{Open access}

Términos de uso

Licencia creative commons

Etica de publicaciones

Revisión por pares

Gestión por Open Journal System DOl: http://dx.doi.org/10.21615/

cesder.11.1.8

ISSN: 2145-7719

\section{Sobre el artículo:}

El presente trabajo fue elaborado a los fines de ser presentado oralmente en el Encuentro Nacional de Investigadoras e Investigadores en Ciencias Jurídicas y Sociales 28-29-30 de agosto 2019, FCJS - UNL.

\section{Sobre los autores:}

1. Doctora en Derecho. Universidad Católica de Santa Fe.

\section{Resumen}

Este trabajo se propone desarrollar una crítica de la noción de paz, como concepto fundante del derecho internacional moderno. Se procura explicar cómo la raigambre liberal de esta noción, condujo a su consolidación como armisticio, como contención de hostilidades vigentes. En primer lugar, se analiza el rol de la Paz de Westfalia, a través del surgimiento del principio de no intervención, en la construcción de la figura del Estado garante de la paz. Luego se presentan los sucesivos aportes significativos por parte de la Sociedad de Naciones y sobre todo de Naciones Unidas a través de la regulación y finalmente prohibición del uso de la fuerza. En particular se analizará la funcionalidad de los principios de no uso de la fuerza y solución pacífica de controversias en la configuración de la idea de paz internacional. Por último, se postula el potencial del reciente concepto de responsabilidad de proteger para la gestación de una auténtica paz.

A través de esta última figura jurídica se propone un enfoque diferencial-relacional del derecho internacional como alternativa para repensar la convivencia internacional en clave plural, para pasar de una paz como armisticio a una auténtica paz como acogida de la alteridad, como hospitalidad.

Palabras Clave: Paz, Derecho internacional, Responsabilidad de proteger.

\section{Abstract}

This paper aims to develop a critical genealogy of the notion of peace, as a founding concept of modern international law. An attempt is made to explain how the liberal roots of this notion led to its consolidation as an armistice, as a containment of existing hostilities. First, the role of the Peace of Westphalia is analyzed, through the emergence of the principle of non-intervention, in the construction of the figure of the State that guarantees peace. Then, the successive contributions that represented the League of Nations and, above all, the United Nations through the regulation and finally prohibition of the use of force are presented. In particular, the functionality of the principles of non-use of force and peaceful resolution of disputes in the configuration of the idea of international peace will be analyzed. Finally, the potential of the recent concept of responsibility to protect for the gestation of genuine 
2. Abogada. Universidad Católica de Santa Fe.

3. Doctora en Derecho. Universidad Católica de Santa Fe.

4. Estudiante de Derecho. Universidad Católica de Santa Fe. peace is postulated. Through this last legal figure, a differential-relational approach to international law is proposed as an alternative to rethink international coexistence in a plural way, to move from a peace as an armistice to a real peace as a host of otherness, as hospitality.

Keywords: Peace, International Law, Responsibility to protect

\section{Introducción}

La guerra es tan antigua como la convivencia social, pero la paz es un invento moderno (Howard, 2001, p. 19). A partir de esta premisa este trabajo se propone esbozar una genealogía de la noción moderna de paz que se institucionaliza a través del Derecho Internacional Público (DIP). ${ }^{1}$

Se postula en primer lugar el carácter liberal de esta paz moderna, en cuanto armisticio que surge de una imposición top-down de la suspensión de la violencia activa. Esta dinámica impositiva pone en evidencia su estrecha vinculación con la lógica hegemónica del Estado Leviatán, en tanto éste supone una naturaleza humana hostil aplacada por el ente supremo regulador (Hobbes, 1980, p. 267). De allí surge, como primer corolario, la íntima relación entre la figura del Estado moderno y el concepto de paz liberal, en tanto paz formal.

En segundo lugar, se procura poner de relieve la inescindible funcionalidad de este concepto liberal de paz a la dinámica regulatoria del sistema que impera en la constelación transnacional (Santos, 1998, p. 19), en cuanto instrumento de consolidación del status quo, de la hegemonía de los países centrales. Ello se realizará a través del rastreo de la traducción normativa del concepto de paz en los instrumentos constitutivos de Sociedad de Naciones y Naciones Unidas.

Finalmente se postula el potencial del concepto de responsabilidad de proteger como portador de un nuevo paradigma en el derecho internacional que puede revertir la lógica liberal, autorreferencial e identitaria del derecho moderno hacia un derecho centrado en la responsabilidad y el pluralismo, un derecho de la hospitalidad como acogida de la alteridad.

\section{La paz de Westfalia}

Con los Tratados de Münster y Ösnabruck, conocidos como Tratados de Paz de Westfalia, se firman en el contexto europeo los primeros compromisos de no intervención intergubernamental. ${ }^{2}$ Con el objetivo de poner fin a las guerras feudales y religiosas de expansión territorial, a través de estos tratados los gobernantes firmantes se obligaban mutuamente a no inmiscuirse en los asuntos internos de los demás gobiernos. ${ }^{3}$ La aceptación de esta obligación implicaba el reconocimiento de la identificación de cada gobernante con un territorio y su población, lo cual da lugar al principio fundante del Estado moderno, el de integridad territorial (Bremer, 2013, p. 23, 30).

Este principio de integridad territorial constituye un primer paso en la conformación del concepto moderno de paz en cuanto ausencia de conflicto armado (Diez de Velasco, 2013, p. 65). Pues el respeto de tal integridad implica la abstención de cualquier interferencia en territorio ajeno, lo cual abarca también todo uso de la fuerza.

1. Sobre la funcionalidad del DIP respecto del aseguramiento de la paz cf. Pagliari, 2004, p. 459

2. Firmados en 1648 estos Tratados ponen fin a los conflictos armados surgidos entre los países del noreste de Europa, conocidos como la Guerra de los Treinta Años. Esta se originó con el objetivo de dejar atrás la estructura política de un imperio unitario fuertemente asentado sobre la religión cristiana coronado por el emperador y el papado y así, dar paso a un nuevo grupo de Estados independientes. Cfr. Bremer, 2013, p. 65.

3. En este sentido, el Art. 64 del Tratado de Ösnabrück, que establecía la relación entre los Estados que conformarían la Confederación de Estados encabezados por Austria, que vendría a suplantar al hasta entonces Sacro Imperio Romano-Germano. Aunque limitado a esos Estados, esta normativa sentó las bases para la gestación de una nueva forma de regulación interestatal. Cf. Bremer, 2013, p. 11 
En vistas a lograr esta integridad territorio-población-poder, comienza con Westfalia un lento proceso de centralización jurídica, que culminará con la diversidad y coexistencia medieval de fuentes a través de la institucionalización del absolutismo jurídico, de un derecho único y hegemónico (cf. Mattei, 2013, p. 29, 31).

De cualquier manera, en Westfalia no queda todavía prohibida explícitamente la guerra, en tanto ésta era entendida como medida de autoprotección, como medio de tutela de los intereses y derechos de los Estados (Acosta Estévez, 2003, p. 17; Bermejo, 2015, p. 218). En efecto, las pretensiones de aseguramiento de los Estados responden a la lógica autorreferencial del derecho moderno centrado en la defensa a ultranza de la Identidad individual del Sujeto de derechos.

Este esquema subjetivista del Estado identitario moderno como regulador de una "sociedad del primero yo" que da lugar a una política del "sálvese quien pueda" (Davy/ Lenzen, 2013, p. 8) se plasma en la narrativa hobbessiana entorno al Estado Leviatán, la cual presupone una sociedad beligerante y una condición humana inexorablemente violenta (Hobbes, 1980, p. 265).

Bajo esta narrativa la figura jurídica del Estado Moderno surge en Europa para establecer una paz de armisticio entre pretensiones expansionistas que, cual fuerzas centrípetas, permanecen siempre en constante tensión (Levinas, 2002, p. 138). Pues la paz del Estado hobbesiano no subvierte la violencia intrínseca del Sujeto soberano autorreferencial. Se trata en cambio de una paz paradójicamente violenta. La paz que asegura el status quo de las identidades hegemónicas, la paz de la tranquilidad del sí con sigo mismo, acontece como autoafirmación, y por lo tanto como indiferencia por la alteridad. Puesto que toda autoafirmación, todo aseguramiento de la autoposición, ocurre a costa de la posición de un otro, que es desplazado de esa posición, que es anulado en su alteridad. Esta paz por tanto anula las diferencias, anula la alteridad (cf. Levinas, 2006, p. 143).

\section{La paz de Versalles y la regulación de la guerra en Sociedad de Naciones}

La paz de Versalles ${ }^{4}$ viene a consolidar la paz liberal esbozada en Westfalia. Particularmente el Artículo 10 del Pacto de Sociedad de Naciones (SN) recoge los principios de integridad territorial e independencia política ${ }^{5}$ como límite a toda agresión exterior a sus miembros.

Aunque no prohibida, la guerra pasa a ser considerada una última instancia, frente a la frustración de alternativas de solución de controversias como el arbitraje, el arreglo judicial o a la intervención del Consejo (Art. 12 Pacto SN). Ello dio lugar al surgimiento de guerras ilícitas que serían aquellas explícitamente reguladas por el Pacto como la guerra de agresión (Art. 10), la emprendida antes de cualquier intento pacífico de solución (Art. 12.1) o contra un Estado que estuviese acatando una decisión judicial o arbitral o las recomendaciones del Consejo (Art. 13.4 y 15.6.) (Bermejo, 2015, p. 222).

4. La firma del Tratado de paz de Versalles (1919), mediante el cual se da nacimiento a la Sociedad de Naciones, surge principalmente como consecuencia del impulso generado por el entonces presidente de Estados Unidos, Woodrow Wilson, quien "creía firmemente en el establecimiento de la paz mediante la creación de una Sociedad de Naciones que garantizase la seguridad de cada uno de sus miembros".

5. Artículo 10 Pacto de la Sociedad de Naciones: "Los miembros de la sociedad se comprometen a respetar y a mantener contra toda agresión exterior la integridad territorial y la independencia política presente de todos los miembros de la sociedad. En caso de agresión, de amenaza o de peligro de agresión, el consejo emitirá opinión sobre los medios de asegurar la ejecución de esta obligación." 
Aunque con el explícito principal objetivo de proteger a sus miembros, y en este sentido con un claro tinte nacionalista, autorreferencial y endogénico, ${ }^{6}$ la regulación del uso de la fuerza en SN significa un avance significativo en la consolidación de la prohibición del uso de la fuerza. Aun cuando en esta primera instancia quede la paz internacional todavía subordinada a la seguridad nacional. ${ }^{7}$

Por fuera del Pacto de SN pueden encontrarse en este período otros antecedentes de la prohibición del uso de la fuerza como el proyecto de Tratado de Asistencia Mutua de Lord Robert Cecil, que consideraba a la guerra de agresión como un crimen internacional (Art. 1) y el Protocolo para el arreglo pacífico de las controversias internacionales, denominado también Protocolo de Ginebra, del 2 de octubre de 1924; ambos tratados en la Asamblea de SN. Fuera del ámbito de SN pueden nombrarse el Pacto Renano de garantía mutua, firmado en el marco de los Acuerdos de Locarno de 1925 firmado entre Alemania, Bélgica, Francia y Gran Bretaña, por el que se comprometían a no recurrir en ningún caso a la guerra, ni a ningún ataque o invasión (Art. 2) y el famoso Pacto Briand-Kellogg (conocido así por los apellidos de sus propulsores, los ministros francés y británico respectivamente) de 1928, por el que sesenta y tres Estados renunciaron explícitamente a la guerra. El fracaso de este último es atribuído a la falta de mecanismos de respuesta frente a las violaciones a la citada prohibición, que dejaría impune a los Estados incumplidores (Cf. Bermejo, 2015, p. 230 ss).

\section{La paz en Naciones Unidas}

La creación de Naciones Unidas $(\mathrm{NU})^{8}$ introdujo por primera vez la prohibición de la guerra como norma imperativa del DIP a través de la institucionalización del principio de no uso de la fuerza (Acosta Estévez, 2003, p. 16). Con una narrativa marcadamente más universalista y el explícito propósito de mantener la paz y la seguridad internacionales (Art. 1 Carta NU) los Estados miembros de la Organización proscribieron de manera vinculante el uso de la fuerza armada en el Artículo 2.4 de su Carta fundacional (Acosta Estévez, 2003, p. 16). ${ }^{9}$

Reafirmando los principios de integridad nacional y soberanía ${ }^{10}$ bosquejados en Westfalia y posteriormente recogidos en el tratado de Versalles, la Carta incorpora el principio de no uso de la fuerza (Art. 2. inc. 4) respaldado por el de solución pacífica de controversias (Art. 2. inc. 3 y Art. 33), que ofrece mecanismos para su concreción.

A los fines de la efectivización de sus propósitos, y para superar la deficiencia de SN respecto de la previsión de instrumentos de garantía de tales propósitos, la ONU puso particular énfasis en afianzar su estructura y mecanismos. En este sentido le atribuye un rol fundamental al Consejo de Seguridad, en tanto responsable primordial del mantenimiento de la paz y seguridad internacionales (Art. 24 Carta NU).

Desde entonces NU ha liderado las principales estrategias internacionales para la consecución de sus propósitos de mantención de la paz y seguridad internacionales,

6. En este sentido el Art. 11 del Pacto de SN: "Se declara expresamente que toda guerra o amenaza de guerra, afecte directamente o no a uno de los miembros de la sociedad, interesa a la sociedad entera y que ésta debe adoptar las medidas adecuadas para salvaguardar eficazmente la paz de las naciones."

7. En este sentido en el Pacto de SN el Art. 8.1: "Los miembros de la sociedad reconocen que el mantenimiento de la paz exige la reducción de los armamentos nacionales al mínimum compatible con la seguridad nacional y con la ejecución de las obligaciones internacionales impuestas por una acción común." y el Art. 15. "En el caso en que el consejo no consiguiera hacer aceptar su informe por todos los miembros, fuera de los representantes de toda parte en la divergencia, los miembros de la sociedad se reservan el derecho de proceder como lo juzgaran necesario para el mantenimiento del derecho y de la justicia."

8. Luego de la segunda guerra mundial, en el año 1945, en la ciudad de San Francisco, se celebró la Conferencia de las Naciones Unidas en la cual 51 estados, mediante la firma del documento fundacional, dieron origen a la Organización de las Naciones Unidas, cuyo propósito principal es el de mantener la paz y la seguridad internacionales y de la cual actualmente 153 países forman parte.

9. Esta prohibición no es sin embargo absoluta, pues el uso de la fuerza puede ser considerado todavía lícito si funciona para repeler una agresión, es decir la guerra es lícita como instrumento para repeler una guerra ilícita, tanto bajo la figura de la legítima defensa que habilitaría a cualquier Estado, así como bajo las atribuciones que la Carta de NU confiere al Consejo de Seguridad. Cf. Carta NU, Cap. VII, Art. 39, 44 y 50.

10. En este sentido, el artículo 2 inciso 7 de la Carta NU: "Ninguna disposición de esta Carta autorizará a las Naciones Unidas a intervenir en los asuntos que son esencialmente de la jurisdicción interna de los Estados, ni obligará; a los Miembros a someter dichos asuntos a procedimientos de arreglo conforme a la presente Carta; pero este principio no se opone a la aplicación de las medidas coercitivas prescritas en el Capítulo VII" 
logrando a su vez un reconocimiento sin precedentes acerca de su imprescindible rol para con tales propósitos, a través de la expansión global de su paradigma liberal de paz.

Aunque son de destacar las medidas al respecto como las relacionadas con el desplazamiento forzado, los refugiados, los menores de edad, o las de erradicación de minas antipersonales, el desarme o las operaciones de mantenimiento de la paz (Cf. Rettberg, 2013, p. 27), no faltan las advertencias acerca de las falencias de la Organización para alcanzar sus fines. En este sentido, ya en 1992, el entonces Secretario General de la Organización, Boutros Boutros-Ghali, reconocía al fin de la Guerra Fría, tal fracaso (1992, n. 2).

También genera ciertas conjeturas respecto de su capacidad para establecer una auténtica paz, la incoherencia que significa el sostenimiento de los cinco miembros permanentes del Consejo de Seguridad y su privilegiada capacidad de veto (Art. 27. inc.3 Carta NU) en concurrencia con un discurso universalista pro-democracia (Rettberg, 2013, p. 28). ${ }^{11}$

En concordancia con ello y siendo el Consejo el que detenta el monopolio de la fuerza (art. 42 Carta NU), resulta cuando menos paradójico que hayan sido tales miembros permanentes los que en el último siglo y en nombre de la paz, hayan utilizado a menudo la fuerza para proteger sus intereses (cf. Bremer, 2013, p. 71).

Esta paradoja refleja la dinámica dual de la identidad hegemónica moderna, la cual hace la guerra para alcanzar su paz. Esta paz de la hegemonía se identifica con el reposo, con el bienestar de quienes detentan el poder. Ello se puede notar en las afirmaciones acerca del relativo éxito de NU, que identifican la disminución de la violencia internacional con el cese de los conflictos en el seno de los países que detentan la hegemonía mundial.

En cambio, en las periferias, cuando no acontece la guerra se vive la cruda violencia de la indiferencia: la violencia que significa el sufrimiento del hambre, de la pobreza, en un mundo en el que hay alimentos para todos, en el que hay lugar para todos.

Sugestivamente advertía el Secretario Boutros-Ghali que para alcanzar la paz era tan importante actuar sobre los "ejércitos hostiles" como "tratar de poner fin a las causas más hondas de los conflictos: la desesperación económica, la injusticia social y la opresión política." (1992, n. 15). Surgida paradójicamente en el seno de NU, puede detectarse en esta advertencia un atisbo de revisión del modelo hegemónico de la paz liberal. En este sentido, tal advertencia tiene potencial para transformar la paz formal del armisticio en una auténtica paz como responsabilidad.

\section{La paz de la responsabilidad de proteger}

Si bien Naciones Unidas contribuyó a la naturalización del concepto de paz como armisticio, ${ }^{12}$ en su seno también han surgido reclamos y alternativas que pueden aportar a la consolidación de una auténtica paz. ${ }^{13}$

11. El Consejo de Seguridad es el órgano de Naciones Unidas encargado de mantener la paz y la seguridad internacionales. Se compone de quince miembros, cinco de los cuales son permanentes: China, Estados Unidos, Rusia, Francia y Reino Unido, mientras que los diez restantes son elegidos por la Asamblea General por períodos de dos años. Cf. Barboza, 2008, p. 311-312; Carrillo Suárez, 20015, p. 57-58.

12. En este sentido, en el año 2006 se crearon en la órbita de Naciones Unidas departamentos encargados de las actividades de consolidación de la paz como la Comisión de Consolidación de la Paz (United Nations Peacebuilding Commission, UNPBC), un Fondo para la Consolidación de la Paz y una Oficina de Apoyo a la Consolidación de la Paz.

13. Aunque este espacio para el disenso y la participación contribuya a mostrar un perfil "democrático" de la Organización, contribuye sin embargo a sostener la hegemonía de los países centrales, que son los que priman al momento de la toma de las decisiones "imperativas." 
En este sentido, el reciente principio de responsabilidad de proteger ${ }^{14}$ encierra el potencial de revisar la lógica absolutista del derecho moderno, al incorporar en el derecho internacional contemporáneo, e incluso en el derecho occidental en general, una nueva impronta ético-relacional. Ello en cuanto la referencia a la alteridad que implica la noción de responsabilidad subvierte la dinámica autorreferencial del derecho identitario moderno.

Técnicamente, el principio de responsabilidad de proteger, habilita la intervención de la comunidad internacional, e hipotéticamente también de los Estados en particular, frente a graves violaciones de los derechos humanos en otro Estado. ${ }^{15}$ Más allá del complejo debate sobre las condiciones de habilitación de tal intervención, así como de la delicada cuestión acerca de su funcionalidad como vía de justificación de un uso justo de la fuerza (cf. Añaños Meza, 2010, p. 200; Piedrahita Ramírez, 2015, p. 65; Espósito, 2005, p. 5), este principio incorpora un cambio de enfoque respecto de la lógica moderna de las subjetividad jurídica. Ello en tanto que pone el acento en los sujetos beneficiarios de la acción, más que en el sujeto interviniente, titular de la responsabilidad (López-Jacoiste, 2006, p. 290). De esta manera incorpora una referencia a un aspecto del derecho que desborda el ámbito seguro de la Identidad subjetiva, - a nivel Estatal, de la integridad nacional -, en virtud de la protección de la alteridad.

En este sentido la responsabilidad de proteger pone el acento en el carácter debitorio del derecho, invirtiendo la lógica autorreferencial del derecho subjetivo como pretensión. La responsabilidad de proteger señala antes que nada un deber que refiere una responsabilidad de todos para con todos. ${ }^{16}$

Repensar el derecho como responsabilidad habilita a repensar la paz como convivencia en las diferencias. Paz no como tranquilidad del sí consigo mismo, sino como tensión, como no-indiferencia (Levinas, 1992, p. 139), como pre-ocupación por los demás.

En este sentido, la responsabilidad de proteger entendida como invocación a una pre-ocupación por los demás, puede contribuír a revisar la dinámica autorreferencial del derecho que regulas la constelación transnacional desde la modernidad, en vistas a la gestación de un derecho transnacional más plural.

\section{Perspectivas: Una agenda para la paz}

El concepto de paz constituye un elemento fundante del derecho moderno. Éste tiene como fin asegurar la paz social interna y externa de los Estados. En efecto, tanto el derecho moderno como su concepto liberal de paz social funcionan ambos en torno a la figura central del Estado Soberano, que es en definitiva en este modelo el garante de la paz (Cf. Kant, 2003, p. 1-2).

Este paradigma de paz liberal que se institucionalizó en el Derecho Internacional desde Westfalia y se expandió globalmente con Naciones Unidas, incluso a fuerza de una importante dosis de violencia (Mattei, 2013, p. 30), contempla ante todo un abordaje de la paz como armisticio, como ausencia de conflicto armado.

14. Este concepto aparece por primera vez el documento "La responsabilidad de proteger" de la Comisión Internacional sobre Intervención y Soberanía de los Estados (dic. 2001) impulsado por el gobierno de Canadá y luego en "Un mundo más seguro: la responsabilidad que compartimos" del Grupo de Alto Nivel sobre las amenazas, los desafíos y el cambio (1/12/2004), los cuales inspiraron el Informe del Secretario General Kofi Annan titulado "Un concepto más amplio de libertad: desarrollo, seguridad y derechos humanos para todos" (A/59/2005, 21/03/2005). Estos textos dieron lugar luego a los 138 a 140 de la Resolución de la Cumbre de 2005 de NU. (Cf. Añaños, 2010, p. 201-202)

15. El n 139 del documento final elaborado por la Cumbre de Naciones Unidas de 2005 refiere a la responsabilidad de la comunidad internacional de recurrir a medios diplomáticos, humanitarios y otros medios pacíficos apropiados en los casos de genocidio, crímenes de guerra, depuración étnica y crímenes de lesa humanidad, instando -incluso- a la Asamblea General a definir esos medios pacíficos apropiados e incorporarlos para el accionar del Consejo de Seguridad.

16. Respecto de la paz como responsabilidad por el otro cf. Levinas, 2006, p. 149; Messina, 2012, p. 146. 
Tal concepto de paz, empero, no tiene en cuenta la violencia generada por la indiferencia frente a las periferias de la hegemonía mundial (Mattei, 2013, p. 41; Fischer-Lescano/Möller, 2012, p. 12). Las diferencias son acalladas, solapadas por el discurso de la paz formal, que procura fijar la posición, procura la seguridad de las identidades que detentan la hegemonía transnacional. Se trata de la violencia que genera la dinámica centrípeta de la identidad, de la hegemonía, que rige el derecho moderno. Esta dinámica tiende a aplazar toda alteridad, toda diferencia, al pretender incluirlas en su lógica o excluirlas a los márgenes de su mismidad. Se trata entonces de la indiferencia frente a la alteridad en cuanto tal, la indiferencia frente a las diferencias.

Esta dinámica conforma la estructura nodal del modelo identitario que subyace al paradigma subjetivista del derecho moderno, en cuanto orden destinado a ejecutar las pretensiones del Sujeto de Derechos, pretendidamente soberano, autónomo y libre, en tanto que propietario.

En cambio, la consolidación de una auténtica paz, como convivencia armónica y responsable en y por las diferencias, implica la gestación de un orden pluralista, de un derecho que tenga en cuenta la diversidad (Bonet de Viola, 2019, p. 352).

Ello significa repensar el derecho ya no sólo y principalmente como orden regulador sino ante todo a partir del potencial emancipador (Santos, 1998, p. 43, 44) que implica su concepción como responsabilidad. Significa revisar la dinámica regulatoria, jerárquica ("desde arriba"), absolutista y mecanicista del derecho moderno para dar lugar a la dinámica emancipadora, participativa, flexible, holística de esta nueva paz social. ${ }^{17}$ Significa también que la narrativa de la seguridad ceda lugar a una nueva narrativa basada en el debate y el disenso (Fischer-Lescano/Teubner, 2006, p. 58), en tanto instrumentos funcionales para la constitución de esta paz plural. Significa a su vez repensar el derecho desde la responsabilidad antes que desde la pretensión. En este sentido, el concepto de responsabilidad de proteger, habilita una revisión del paradigma subjetivista de la pretensión para pensar los derechos como derechos del otro (cf. Levinas, 2002, p. 137).

La inseguridad que genera, en primer lugar para los actores del derecho, esta flexibilidad, esta deconstrucción del derecho "seguro",18 constituye el primer costo de la apertura a la diversidad, a las diferencias, el primer costo de la pluralidad. Puesto que la apertura a la alteridad en cuanto tal implica el riesgo de salir del ámbito seguro de la hegemonía, de la propia racionalidad.

Esta apertura a la alteridad, a las diferencias, a lo diverso en cuanto tal habilita una vía para pensar el derecho ya no como regulador de la hostilidad sino como gestor de la hospitalidad (Penchaszadeh, 2011, p. 258; Messina, 2012, p. 158). Abre la posibilidad de repensar el orden del derecho como instrumento para una nueva paz social, más auténtica, más solidaria, aunque menos segura, menos tranquila, menos autorreferencial.

La paz de la hospitalidad aquí referida significa la paz de la acogida de la alteridad (Messina, 2012, p. 146). Se trata de una paz tensa, incómoda para la identidad hegemónica, puesto que pone en cuestión toda pretensión de dominio e imposición. Esta paz que escapa a todo orden, en tanto dominación, funciona en para con el derecho como constante herramienta de revisión de las hegemonías (Derrida, 2001, p. 51).

17. Respecto de esta dinámica recesiva cf. Mattei, 2013, p. 109 ss.

18. Sobre la deconstrucción del derecho hegemónico en tanto derecho "seguro" para sus actores cf. Kennedy, 2010, p. 86 y p. 131 
Es por ello que esta paz no pretende plantearse como nuevo orden ni como mecanismo alternativo de establecimiento de una nueva paz. Esta paz plural funciona tan sólo como mecanismo de revisión constante de cualquier orden instituido, funciona como herramienta de resignificación del espacio simbólico que significa el derecho, en cuanto ámbito esencial en toda convivencia plural.

\section{Referencias}

Acosta Estévez, J. B. (2003). El Derecho Internacional ante el fenómeno bélico: la prevención y atenuación de las consecuencias de los conflictos armados. Anuario Mexicano de Derecho Internacional, III, 11-65.

Añaños Meza, C. (2010). La "Responsabilidad de proteger" en Naciones Unidas y la doctrina de la "Responsabilidad de proteger." Anuario Mexicano de Derecho Internacional, 10, 199-244.

Barboza, J. (2008). Derecho Internacional Público (2da. Edición). Buenos Aires: Zavalia.

Bermejo, R. (2015). El uso de la fuerza, la Sociedad de Naciones y el Pacto Briand-Kellogg. En Y. Gamarra Chopo \& C. R. Fernández Liesa (Eds.), Los orígenes del Derecho internacional contemporáneo. Estudios conmemorativos del Centenario de la Primera Guerra Mundial: Vol. II (pp. 217-233). IFC.

Bonet de Viola, A. M. (2019). De la garantía de la identidad a la acogida de las diferencias. Por una Constitución en constante reforma. Revista de Derecho Público. 25 Años de la reforma constitucional de 1994-I, Rubinzal-Culzoni, 327-357.

Bosé, M., \& Fernández Puyana, D. (2017). La Historia de la Paz en Occidente. San José, Costa Rica: UNESCO, University for Peace.

Boutros-Ghali, Boutros. (1992). An Agenda for Peace: Preventive. Diplomacy, Peacemaking and Peace-Keeping (A/47/277-S/24111). Naciones Unidas.

Bremer, J. J. (2013). De Westfalia a post-Westfalia. Hacia un nuevo orden internacional. Mexico: UNAM. Retrieved from https://biblio.juridicas.unam.mx/bjv/detalle-libro/3366-de-westfalia-a-post-westfalia-hacia-un-nuevo-orden-internacional

Carrillo Suárez, A. E. (2015). Curso de Actualización de Profesores de Derecho Internacional Privado. Organismos Internacionales. Mexico: UNAM. Retrieved from https://biblio.juridicas.unam.mx/bjv/detalle-libro/4056-curso-de-actualizacion-de-profesores-de-derecho-internacional-privado-seminario-de-derecho-internacional-facultad-de-derecho

Chandler, D. (2004). The Responsibility to Protect? Imposing the 'Liberal Peace.' International Pacekeeping, 11(1), 59-81. https://doi.org/10.1080/1353331042000228454

Cohrs, P. O. (2018). "Pax Americana": the United States and the transformation of the 20th century's global order. Revista Brasileira de Política Internacional, 61(2). https://doi.org/10.1590/0034-7329201800202

Davy, U., y Lenzen, M. (2013). Einleitung: Demokratie morgen. En U. Davy y M. Lenzen (Eds.), Demokratie morgen.Überlegungen aus Wissenschaft und Politik (pp. 7-15). Bielefeld: Transcript. 
Derrida, J. (2001). ¡Palabra! Instantáneas filosóficas. Madrid: Trotta.

Diez de Velasco, M. (2013). Instituciones del Derecho Internacional Público. Madrid, España: TecnosMa.

Espósito, C. (2005). Uso de la fuerza y responsabilidad de proteger: El debate sobre la reforma de la ONU. Madrid. Retrieved from http://www.almendron.com/politica/ pdf/2005/int/int 1219.pdf

Fischer-Lescano, A., \& Möller, K. (2012). Der Kampf um globale soziale Rechte: Zart wäre das Gröbste. Berlin: Wagenbach Verlag.

Hobbes, T. (1980). Leviatán. (C. Moya \& A. Escohotado, Eds.). Madrid: Editorial Nacional.

Howard, M. (2000). La invención de la paz. Reflexiones sobre la guerra y el orden internacional. España: Salvat.

Kant, I. (2003). La paz perpetua, Biblioteca Universal Virtual, Editorial del Cardo, https://www.biblioteca.org.ar/libros/89929.pdf

Kennedy, D. (2010). Izquierda y Derecho. Ensayos de teoría jurídica crítica. Buenos Aires: Siglo Veintiuno Editores.

Levinas, E. (2006). Paz y proximidad. Revista Laguna, (18), 143-151.

Levinas, E. (2002). Fuera del sujeto. Madrid: Caparrós Editores.

López-Jacoiste Díaz, E. (2006). La responsabilidad de proteger: reflexiones sobre su fundamento y articulación. Anuario Español de Derecho Internacional, (22), 285-315.

Macpherson, C. B. (2005). La teoría política del individualismo posesivo. De Hobbes a Locke. Madrid, España: Trotta.

Mattei, U. (2013). Bienes Comunes. Un Manifiesto. Madrid, España: Trotta.

Messina, A. L. (2012). La Paz como primer lenguaje. Paz y politica En E. Levinas. Ideas y Valores, 61(150), 145-167.

Pagliari, A. S. (2004). El derecho internacional público. Funciones, fuentes, cumplimiento y la voluntad de los Estados. . Anuario Mexicano de Derecho Internacional, IV, 457-473.

Penchaszadeh, A. P. (2011). Política, don y hospitalidad en el pensamiento de Jacques Derrida. Isegoría, Revista de Filosofia Moral y Politica, 44(enero-junio), 257-271.

Piedrahita Ramírez, L. F. (2015). La soberanía como responsabilidad y los fundamentos del nuevo intervencionismo humanitario. Estudios de Filosofía, 51(51), 45-74. https:// doi.org/10.17533/udea.ef.n51.a04

Rettberg, A. (2013). La construcción de la paz bajo la lupa: una revisión de la actividad y de la literatura académica internacional. Estudios Políticos, 42, 13-36. 
Revilla Montoya, P. C. (2010). Hacia la responsabilidad de proteger. Bases jurídicas para una respuesta colectiva ante crisis humanitarias. Anuario Mexicano de Derecho Internacional, VII, 643-673. Retrieved from http://www.ojs.unam.mx/index.php/ amdi/article/view/16054

Sousa Santos, B. de. (1998). La Globalización del Derecho: Los nuevos caminos de la regulación y la emancipación. Santa Fe de Bogotá: Universidad Nacional de Colombia. 